\title{
An Improved Multiple-Toeplitz Matrices Reconstruction Algorithm for DOA Estimation of Coherent Signals
}

\author{
Bingbing $Q I^{1}$, Wei $L I^{2}$ \\ ${ }^{1}$ Dept. of Electronic and Information Eng., Nanjing University of Aeronautics and Astronautics, Nanjing, 211106, China \\ ${ }^{2}$ The 3rd Research Institute of China Electronics Technology Group Corporation, Beijing, 100015, China
}

13699160512@163.com

Submitted January 21, 2021 / Accepted May 7, 2021

\begin{abstract}
The Toeplitz matrix reconstruction algorithms exploit the row vector of an array output covariance matrix to reconstruct Toeplitz matrix, which provide the directionof-arrival (DOA) estimation of coherent signals. However, the Toeplitz matrix reconstruction method based on any row vector of the array output covariance matrix suffers from signal correlation, it results in poor robustness. The methods based on multi-row vectors suffer serious performance degradation when in the low signal-to-noise ratio $(S N R)$ owing to the noise energy is the square of the input noise energy. To solve the above problems, we propose an improved method that exploits all rows of the time-space correlation matrix to reconstruct the Toeplitz matrix, namely TS-MTOEP. This method firstly uses the coherence of the narrowband signal and the uncorrelated noise at different snapshots to construct the time-space correlation matrix, it effectively eliminates the influence of noise. Then, the Toeplitz matrix is reconstructed via all rows of the time-space correlation matrix, which effectively improves the energy of the signal, and further results in the improvement of the SNR. Finally, the DOAs can be obtained by combining it with the subspace-based methods. The theoretical analysis and simulation results indicate that compared with the existing Toeplitz and spatial smoothing methods, the proposed method in this paper provides good performance on estimation and resolution in cases with low input signal-to-noise due to time-space correlation matrix processing. Furthermore, in cases where the DOAs between the coherent sources are closely spaced and the snapshot number is low, our proposed method significantly improves the performance of the DOA estimation. We also provide the code to realize the reproducibility of the proposed method.
\end{abstract}

\section{Keywords}

Coherent signals, DOA estimation, subspace-based methods, time-space correlation matrix, Toeplitz reconstruction

\section{Introduction}

Direction-of-arrival (DOA) estimation is a major research issue in array signal processing and has been widely used in radar, sonar, navigation and wireless communication [1], [2]. Many subspace-based algorithms including MUSIC (Multiple Signal Classification) [3] and ESPRIT (Estimation of Signal Parameters by Rotational Invariance Techniques) [4] have the disadvantage of rank loss of the signal covariance matrix in cases where the signals are coherent. This leads to a low DOA estimation performance or even the failure of those algorithms. To process the coherent signals, researchers proposed the forward spatial smoothing algorithm (FOSS) [5] and the forward/backward spatial smoothing algorithm (FBSS) [6]. The spatial smoothing technique effectively resolves the coherent signals, however, it reduces the effective aperture of the array and decreases the DOA estimation performance. Although the subspace fitting [7] and the compressed sensing algorithms [8] are not affected by signal coherence and can directly estimate the parameters of coherent signals, however, they require a high computational complexity.

ESPRIT-like method [9] and its variants have also received considerable attentions due to their low computational complexity. The ESPRIT-like method [9] exploits any single row of the covariance matrix of an array output covariance matrix to reconstruct the Toeplitz matrix. However, it is affected by the phase difference and incident angle of the coherent signals, which leads to degradation of the DOA estimation performance or even failure in some cases. To solve the robustness problem of ESPRIT-like algorithm, Zhang et al. [10] proposed multiple Toeplitz matrix reconstruction (MTOEP) method and forward/backwards partial Toeplitz reconstruction (FBPTMR) method [11], respectively. These methods in [10] and [11] use the square summation of several rows of the covariance matrix of the array output to reconstruct Toeplitz matrix. This leads to the fact that the noise energy is the square of input noise energy and therefore the DOA estimation performance is relatively low at low signal-tonoise ratio (SNR). 
Many other matrix reconstruction methods based on higher-order cumulant [12-14] have been proposed to resolve coherent signals in the low SNR region. Unfortunately, these methods require large numbers of snapshots and suffer from burdensome computation. The spatial differencing methods [15-17] can also to achieve the DOA estimation of coherent signals. However, the DOAs of coherent and uncorrelated signals are estimated separately, which increased the computational complexity, and reduced the DOA estimation performance of the uncorrelated signals.

To overcome the above-mentioned issue, in this paper we propose an improved Multiple-Toeplitz matrices reconstruction method, namely TS-MTOEP. The proposed method uses the strong correlation of signal and the weak correlation of noise in time and space domains to construct a time-space correlation matrix. Then, based on the timespace correlation matrix, the MTOEP method is adopted to reconstruct the Toeplitz matrix. Finally, the DOAs can be directly obtained using the subspace method. The theoretical and simulation results indicate that the proposed method enhances the noise suppression ability and improves the DOA estimation performance. The main contributions of the proposed method are as follows:

1) The proposed method based on the use of strong correlation of signal and the weak correlation of noise at different snapshots in time and space domains to construct the time-space correlation matrix, which effectively eliminates the influence of the noise.

2) The Toeplitz matrix reconstruction based on the complete information of the time-space correlation matrix via the MTOEP method to restore the rank and improves the SNR, which produces better DOA estimation performance at low SNRs than those Toeplitz matrix reconstruction methods based on the array output covariance matrix and the classical spatial smoothing method.

3) Compared with MTOEP method, the proposed method has relatively less computational complexity.

The organization of this paper is as the following. We present the signal model in Sec. 2. The problem formulation is described in Sec. 3. Then in Sec. 4, the proposed method based on time-space processing is introduced, followed by simulation results and discussions in Sec. 5 . Finally, this paper is concluded in Sec. 6.

Symbols: matrices, vectors and scalars are represented by capital bold letters, lower-case bold letters and lower-case letters, respectively. $(\cdot)^{\mathrm{H}},(\cdot)^{\mathrm{T}}$ and $(\cdot)^{*}$ represent conjugate transpose, transpose, and conjugate, respectively.

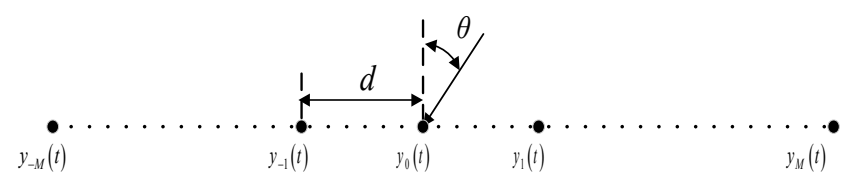

Fig. 1. Antenna array model.
$E[\cdot]$ represents mathematical expectation, $\boldsymbol{0}_{m \times n}, \boldsymbol{I}_{m}$ and $\operatorname{diag}\{\cdot\}$ represent $m \times n$ zero matrix, $m \times m$ unit matrix and diagonal matrix, respectively.

\section{Signal Model}

Consider there are $P$ narrowband far-field sources impinged on a linear uniform array (ULA) composed of $N=2 M+1$ elements with equal spacing from distinct directions $\theta_{1}, \cdots, \theta_{P},(P<M)$.

Let the array element with index 0 be the reference, the complex envelope of each narrowband signal at the reference element be $s_{i}(t), i=1,2, \ldots, P$, and the spacing between the adjacent array elements be $d=\lambda / 2$, where $\lambda$ is the carrier wavelength.

We further assume that the first $L$ signals are mutually coherent and the other $P-L$ signals are uncorrelated and independent of the first signal. Let the first signal $s_{1}(t)$ be the reference, then the $k$-th coherent signal $s_{k}(t)$ can be expressed as

$$
s_{k}(t)=\beta_{k} \mathrm{e}^{\mathrm{j} \Delta \psi_{k}} s_{1}(t), k=1, \cdots, L
$$

where $\beta_{k}$ is the amplitude fading factor, and $\Delta \psi_{k}$ denotes the phase difference of $s_{k}(t)$ related to $s_{1}(t)$ (without loss of generality, we assume $\left.\beta_{1}=1, \Delta \psi_{1}=0\right)$.

The received data vector by the array at time $t$ is

$$
\begin{aligned}
\boldsymbol{y}(t) & =\left[y_{-M}(t), \cdots, y_{0}(t), \cdots, y_{M}(t)\right]^{\mathrm{T}} \\
& =\boldsymbol{x}(t)+\boldsymbol{n}(t)=\boldsymbol{A} \boldsymbol{s}(t)+\boldsymbol{n}(t)
\end{aligned}
$$

where $\boldsymbol{A}=\left[\boldsymbol{a}\left(\theta_{1}\right), \cdots, \boldsymbol{a}\left(\theta_{P}\right)\right]$ denotes the $(2 M+1) \times P$ array steering matrix with the steering vector $\boldsymbol{a}\left(\theta_{P}\right)=\left[\mathrm{e}^{-\mathrm{j} 2 \pi / \lambda M d \sin \theta_{P}}, \cdots, 1, \cdots, \mathrm{e}^{\mathrm{j} 2 \pi / \lambda M d \sin \theta_{P}}\right]^{\mathrm{T}}$ of the $i$-th signal. $\boldsymbol{s}(t)=\left[s_{1}(t), \cdots, s_{P}(t)\right]^{\mathrm{T}}$ represents the $P \times 1$ source signal vector. $\boldsymbol{n}(t)=\left[n_{-M}(t), \cdots, n_{M}(t)\right]^{\mathrm{T}}$ is the Gaussian white noise vector assumed to be uncorrelated to signals with zero mean and variance $\sigma_{n}^{2}$.

For the far-field narrowband signals, the $i$-th signal $s_{i}(t)$ has the following expression

$$
s_{i}(t)=v_{i}(t) \exp \left((\mathrm{j} \omega t)+\varphi_{i}(t)\right)
$$

where $\omega, v_{i}(t)$, and $\varphi_{i}(t)$ are the signal carrier frequency, signal instantaneous envelope and the signal instantaneous phase, respectively. Both $v_{i}(t)$ and $\varphi_{i}(t)$ are the narrowband low frequency signal with slow changes relative to the carrier frequency $\omega$.

As described in (2), $\boldsymbol{n}(t)$ is an additive noise vector whose elements as stationary, spatially and temporally white, zero-mean random processes that are independent of the source signals. That is 


$$
\begin{gathered}
E\left[\boldsymbol{n}(t+\tau) \boldsymbol{n}^{\mathrm{H}}(t)\right]=\sigma_{n}^{2} \delta(\tau) \boldsymbol{I}, \\
E\left[\boldsymbol{n}(t+\tau) \boldsymbol{n}^{\mathrm{H}}(t)\right]=\boldsymbol{0}, \text { for any } \tau
\end{gathered}
$$

where $\delta(\tau)$ is the delta function, $\boldsymbol{I}$ denotes the identity matrix, $\sigma_{n}^{2}$ is the noise power at each sensor. The noise characteristics described here are used in time-frequency domain methods [18-20] to improve DOA estimation performance.

Based on the above assumptions, the array output covariance matrix is expressed as

$$
\boldsymbol{R}_{\mathrm{yy}}=E\left[\boldsymbol{y}(t) \boldsymbol{y}^{\mathrm{H}}(t)\right]=\boldsymbol{A} \boldsymbol{R}_{\mathrm{S}} \boldsymbol{A}^{\mathrm{H}}+\sigma_{n}^{2} \boldsymbol{I}_{N}
$$

where $\boldsymbol{R}_{\mathrm{S}}=E\left[\boldsymbol{s}(t) \boldsymbol{s}^{\mathrm{H}}(t)\right]$ represents the signal covariance matrix.

For the DOA estimation of narrowband far-field source, the impinged source and noise are stationary random process, which results in the received data $y_{i}(t)$, $i=-M, \ldots, M$ also to be a random process. Therefore, it is necessary to exploit the characteristics of the received data in a statistical sense, which has been widely used in the DOA estimation methods [21-23].

It can be seen from (2) that the data received by different array elements all contain signal source information, that is, they appear with equal probability, the Mathematical Expectation could be simplified to the time average. Thus, in practice, the array output covariance matrix $\boldsymbol{R}_{\mathrm{yy}}$ and signal covariance matrix $\boldsymbol{R}_{\mathrm{S}}$ are unknown, these matrices can be estimated with finite number of snapshots

$$
\hat{\boldsymbol{R}}_{\mathrm{yy}}=\frac{1}{K} \sum_{t=1}^{K} \boldsymbol{y}(t) \boldsymbol{y}^{\mathrm{H}}(t), \hat{\boldsymbol{R}}_{\mathrm{S}}=\frac{1}{K} \sum_{t=1}^{K} \boldsymbol{s}(t) \boldsymbol{s}^{\mathrm{H}}(t) .
$$

\section{Problem Formulation}

We use the data vector $\boldsymbol{y}(t)$ to construct an $(M+1) \times(M+1)$ Toeplitz matrix

$$
\begin{aligned}
& \boldsymbol{R}_{\mathrm{Y}}(t) \\
= & {\left[\begin{array}{cccc}
y_{0}(t) & y_{1}(t) & \cdots & y_{M}(t) \\
y_{-1}(t) & y_{0}(t) & \cdots & y_{M-1}(t) \\
\vdots & \vdots & \ddots & \vdots \\
y_{-M}(t) & y_{-M+1}(t) & \cdots & y_{0}(t)
\end{array}\right] } \\
= & {\left[\begin{array}{cccc}
x_{0}(t) & x_{1}(t) & \cdots & x_{M}(t) \\
x_{-1}(t) & x_{0}(t) & \cdots & x_{M-1}(t) \\
\vdots & \vdots & \ddots & \vdots \\
x_{-M}(t) & x_{-M+1}(t) & \cdots & x_{0}(t)
\end{array}\right]+\left[\begin{array}{cccc}
n_{0}(t) & n_{1}(t) & \cdots & n_{M}(t) \\
n_{-1}(t) & n_{0}(t) & \cdots & n_{M-1}(t) \\
\vdots & \vdots & \ddots & \vdots \\
n_{-M}(t) & n_{-M+1}(t) & \cdots & n_{0}(t)
\end{array}\right] } \\
= & \boldsymbol{R}_{\mathrm{X}}(t)+\boldsymbol{R}_{\mathrm{N}}(t) .
\end{aligned}
$$

Here $\boldsymbol{R}_{\mathrm{X}}(t)$ represents the Toeplitz matrix constructed by the received signal vector $\boldsymbol{x}(t), \boldsymbol{R}_{\mathrm{N}}(t)$ denotes the Toeplitz matrix constructed by the noise vector $\boldsymbol{n}(t)$.
Therefore, the correlation matrix between $\boldsymbol{R}_{\mathrm{Y}}(t)$ and the obtained data $y_{i}(t)$ from the $i$-th array element, i.e., ESPRIT-like algorithm [9]. Using the result in [9], the reconstructed Toeplitz matrix via any row of the array output covariance matrix can be expressed as

$$
\begin{aligned}
\boldsymbol{R}_{\mathrm{Y} i}(t) & =E\left[\boldsymbol{R}_{\mathrm{Y}}(t) y_{i}^{*}(t)\right]=\tilde{\boldsymbol{A}} \tilde{\boldsymbol{S}}_{i} \tilde{\boldsymbol{A}}^{\mathrm{H}}+\sigma_{n}^{2} \tilde{\boldsymbol{I}}_{M+1, i} \\
& =\boldsymbol{R}_{\mathrm{X} i}(t)+\sigma_{n}^{2} \tilde{\boldsymbol{I}}_{M+1, i}
\end{aligned}
$$

where $\tilde{\boldsymbol{A}}(t)=\left[\tilde{\boldsymbol{a}}\left(\theta_{1}\right), \cdots, \tilde{\boldsymbol{a}}\left(\theta_{P}\right)\right]$ denotes the $(M+1) \times P$ array steering matrix with the $i$-th signal steering vector as $\tilde{\boldsymbol{a}}\left(\theta_{P}\right)=\left[1, \cdots, \mathrm{e}^{-\mathrm{j} 2 \pi / \lambda M d \sin \theta_{P}}\right]^{\mathrm{T}} . \tilde{\boldsymbol{I}}_{M+1, i}$ is an $(M+1) \times(M+1)$ matrix with one on the $i$-th diagonal and zero elsewhere. $\tilde{\boldsymbol{S}}_{i}=E\left[\boldsymbol{S}(t) x_{i}^{*}(t)\right]$ represents the correlation matrix between $\boldsymbol{S}(t)$ and the obtained signal $x_{i}(t)$ from the $i$-th element, where the signal diagonal matrix. can be expressed as $\boldsymbol{S}(t)=\operatorname{diag}\left\{s_{1}(t), \cdots, s_{P}(t)\right\}$.

To further improve the utilization rate of the array elements, Zhang et al. [10] proposed an MTOEP algorithm by exploiting the square summation of $\boldsymbol{R}_{\mathrm{Y} i}(t), i \in[-M, M]$ to reconstruct the Toeplitz matrix. Using the result in [10], the reconstructed Toeplitz matrix via all information of the array output covariance matrix can be expressed as

$$
\begin{aligned}
& \boldsymbol{R}_{\mathrm{METOP}}= \sum_{i=-M}^{M} \boldsymbol{R}_{\mathrm{Y} i}(t) \boldsymbol{R}_{\mathrm{Y} i}^{\mathrm{H}}(t) \\
&= \sum_{i=-M}^{M}\left(\boldsymbol{R}_{\mathrm{X} i}(t)+\sigma_{n}^{2} \tilde{\boldsymbol{I}}_{M+1, i}\right)\left(\boldsymbol{R}_{\mathrm{X} i}(t)+\sigma_{n}^{2} \tilde{\boldsymbol{I}}_{M+1, i}\right)^{\mathrm{H}} \\
&= \tilde{\boldsymbol{A}}\left(\sum_{i=-M}^{M} \tilde{\boldsymbol{S}}_{i} \tilde{\boldsymbol{A}}^{\mathrm{H}} \tilde{\boldsymbol{A}} \tilde{\boldsymbol{S}}_{i}^{\mathrm{H}}+2 \sigma_{n}^{2} \sum_{k=0}^{M} \boldsymbol{D}^{k} \boldsymbol{R}_{S}\left(\boldsymbol{D}^{k}\right)^{\mathrm{H}}\right) \tilde{\boldsymbol{A}}^{\mathrm{H}} \\
& \quad+(M+1) \sigma_{n}^{4} \boldsymbol{I}_{M+1}, \\
& \boldsymbol{D}=\operatorname{diag}\left\{\mathrm{e}^{\mathrm{j} \frac{2 \pi}{\lambda} d \sin \theta_{1}}, \mathrm{e}^{\mathrm{j} \frac{2 \pi}{\lambda} d \sin \theta_{2}}, \cdots, \mathrm{e}^{\mathrm{j} \frac{2 \pi}{\lambda} d \sin \theta_{P}}\right\} .
\end{aligned}
$$

To overcome the issue with the robustness of ESPRIT-like method affected by phase difference and incident angle of the coherent signals, Zhang et al. [11] also proposed an FB-PTMR algorithm by using the square summation of $\boldsymbol{R}_{\mathrm{Y} i}(t), i \in[0, M]$ to reconstruct Toeplitz matrix. Using the result in [11], the reconstructed Toeplitz matrix via half rows of the array output covariance matrix can be written as

$$
\begin{aligned}
& \boldsymbol{R}_{\mathrm{FB}-\mathrm{PTMR}}=\sum_{i=0}^{M} \boldsymbol{R}_{\mathrm{Y} i}(t) \boldsymbol{R}_{\mathrm{Y} i}^{\mathrm{H}}(t) \\
& =\tilde{\boldsymbol{A}}\left(\sum_{i=0}^{M} \tilde{\boldsymbol{S}}_{i} \tilde{\boldsymbol{A}}^{\mathrm{H}} \tilde{\boldsymbol{S}}_{i}^{\mathrm{H}}\right) \tilde{\boldsymbol{A}}^{\mathrm{H}} \\
& \quad+\sigma_{n}^{2} \sum_{i=0}^{M} \tilde{\boldsymbol{A}} \tilde{\boldsymbol{S}}_{i} \tilde{\boldsymbol{A}}^{\mathrm{H}} \tilde{\boldsymbol{I}}_{M+1, i}+\sigma_{n}^{2} \sum_{i=0}^{M} \tilde{\boldsymbol{I}}_{M+1, i}^{\mathrm{H}} \tilde{\boldsymbol{A}} \tilde{\boldsymbol{S}}_{i}^{\mathrm{H}} \tilde{\boldsymbol{A}}^{\mathrm{H}} \\
& \quad+\sigma_{n}^{4} \operatorname{diag}\{M+1, M, \cdots, 1\} .
\end{aligned}
$$

From (10) and (12), we can see that the noise energy in the FB-PTMR and MTOEP methods is the square of the 
input noise energy. This results in decreasing the noise suppression ability and reduces the DOA estimation performance at the low SNR values. Moreover, the FB-PTMR method introduces a color noise, which causes the eigenvalues of the signal covariance matrix deviated from the eigenvalue distribution in the white-noise environment. This further reduces the DOA estimation performance at the low SNR values. Compared with the FB-PTMR algorithm, the MTOEP algorithm doesn't introduce color noise, and results in a higher DOA estimation performance.

\section{The Proposed Method}

To improve the performance of DOA estimation at the low SNR values, here we use the fact that the correlation of noise is weaker than that of the signal in the time and space domains at different snapshots. Then we propose a Toeplitz matrix reconstruction method based on the time-space correlation matrix, which reduces the impact of noise and further improves the DOA estimation performance at the low SNR values.

\subsection{DOA Estimation Based on the Time-space Correlation matrix}

First, similar to (9) and the characteristics of noise as shown in (4), (5), we construct a time-space correlation matrix through the correlation between $\boldsymbol{R}_{\mathrm{Y}}(t)$ and the obtained data $y_{i}(t-\tau)$ from the $i$-th element

$$
\begin{aligned}
\tilde{\boldsymbol{R}}_{\mathrm{Y} i}(t) & =E\left[\boldsymbol{R}_{\mathrm{Y}}(t) y_{i}^{*}(t-\tau)\right] \\
& =E\left[\boldsymbol{R}_{\mathrm{X}}(t) x_{i}^{*}(t-\tau)\right]+E\left[\boldsymbol{R}_{\mathrm{N}}(t) n_{i}^{*}(t-\tau)\right] \\
& =\tilde{\boldsymbol{A}} E\left[\boldsymbol{S}(t) x_{i}^{*}(t-\tau)\right] \tilde{\boldsymbol{A}}^{\mathrm{H}} .
\end{aligned}
$$

Note that as defined in (2) and combining the results of (4), (5). Therefore (13) is reduced to

$$
\tilde{\boldsymbol{R}}_{\mathrm{Y} i}(t)=\tilde{\boldsymbol{A}} E\left[\boldsymbol{S}(t) x_{i}^{*}(t-\tau)\right] \tilde{\boldsymbol{A}}^{\mathrm{H}} .
$$

Then, according the result from (3), when the number of snapshots is small, $v_{i}(t)$ and $\varphi_{i}(t)$ can be considered approximately as constant within these snapshots, and the $i$-th signal $s_{i}(t)$ can be expressed as [20]

$$
\begin{aligned}
s_{i}(t-\tau) & =v_{i}(t-\tau) \exp \left(\mathrm{j} \omega(t-\tau)+\varphi_{i}(t-\tau)\right) \\
& \approx v_{i}(t) \exp \left(\mathrm{j} \omega(t-\tau)+\varphi_{i}(t)\right) \approx s_{i}(t) \mathrm{e}^{-\mathrm{j} \omega \tau}
\end{aligned}
$$

where $\omega$ is the carrier frequency of the narrowband farfield signals.

Equation (15) shows that each signal source has approximately phase difference under a relatively small number of snapshots. It also means that the signal is coherent between different snapshots.

According to the results from (4), (5) and (15), we can find that under a small number of snapshots, the signal has strong correlation and the noise has weak correlation, so this property can be used to improve the DOA estimation performance.

Next, substituting (15) into (14) and combining the result of (9), the time-space correlation matrix $\tilde{\boldsymbol{R}}_{\mathrm{Y} i}(t)$ is obtained as

$$
\begin{aligned}
\tilde{\boldsymbol{R}}_{\mathrm{Y} i}(t) & =\tilde{\boldsymbol{A}} E\left[\boldsymbol{S}(t) x_{i}^{*}(t-\tau)\right] \tilde{\boldsymbol{A}}^{\mathrm{H}} \\
& \approx \tilde{\boldsymbol{A}} E\left[\boldsymbol{S}(t) x_{i}^{*}(t)\right] \mathrm{e}^{-\mathrm{j} \omega \tau} \tilde{\boldsymbol{A}}^{\mathrm{H}} \\
& \approx \tilde{\boldsymbol{A}} \tilde{\boldsymbol{S}}_{i} \mathrm{e}^{-\mathrm{j} \omega \tau} \tilde{\boldsymbol{A}}^{\mathrm{H}} .
\end{aligned}
$$

After that, we substitute (16) into (10), and obtain the Toeplitz reconstruction matrix based on the time-space correlation matrix as

$$
\begin{aligned}
\tilde{\boldsymbol{R}}_{\mathrm{TS}-\mathrm{MTOEP}} & \approx \sum_{i=-M}^{M} \tilde{\boldsymbol{R}}_{\mathrm{Y} i}(t) \tilde{\boldsymbol{R}}_{\mathrm{Y} i}^{\mathrm{H}}(t) \\
& \approx \sum_{i=-M}^{M}\left(\tilde{\boldsymbol{A}} \tilde{\boldsymbol{S}}_{i} \mathrm{e}^{-\mathrm{j} \omega \tau} \tilde{\boldsymbol{A}}^{\mathrm{H}}\right)\left(\tilde{\boldsymbol{A}} \tilde{\boldsymbol{S}}_{i} \mathrm{e}^{-\mathrm{j} \omega \tau} \tilde{\boldsymbol{A}}^{\mathrm{H}}\right)^{\mathrm{H}} \\
& \approx \tilde{\boldsymbol{A}}\left(\sum_{i=-M}^{M} \tilde{\boldsymbol{S}}_{i} \tilde{\boldsymbol{A}}^{\mathrm{H}} \tilde{\boldsymbol{A}} \tilde{\boldsymbol{S}}_{i}^{\mathrm{H}}\right) \tilde{\boldsymbol{A}}^{\mathrm{H}} .
\end{aligned}
$$

By facilitating (17) of the spatial smoothing technique to improve the estimation performance

$$
\tilde{\boldsymbol{R}}_{\mathrm{TS}-\mathrm{MTOEP}}=\tilde{\boldsymbol{R}}_{\mathrm{TS}-\mathrm{MTOEP}}+\boldsymbol{J}\left(\tilde{\boldsymbol{R}}_{\mathrm{TS}-\mathrm{MTOEP}}\right)^{*} \boldsymbol{J}
$$

where $\boldsymbol{J}$ denotes the exchange matrix with ones on its antidiagonal and zeros elsewhere.

Finally, the DOAs are obtained by combining (18) with the subspace-based methods.

It can be concluded from (10) and (17) that the proposed method exploits the strong correlation of the narrowband signal and the weak correlation of the noise at different snapshots to construct the time-space correlation matrix, which effectively eliminates the influence of the noise. Meanwhile, it employs the complete information of the time-space correlation matrix to reconstruct the Toeplitz matrix through the MTOEP method to restore the rank of the covariance matrix and improves the SNR, which produces better DOA estimation performance at the low SNRs.

\subsection{Pseudocode of TS-MTOEP Method}

For the readers' convenience, detailed steps for implementing our proposed method, which is called TSMTOEP, are given as follows

1) The time-space correlation matrix is obtained based on (16).

2) The reconstructed Toeplitz matrix is obtained via (17).

3) Obtain the forward/backward smoothed matrix based on (18). 
4) Estimate the DOAs of sources by combining (18) with TLS-ESPRIT method.

For reproducible research, the software can be obtained at the following website:

https://github.com/qibingbing870612/Coherent-signal-.git

\subsection{Analysis of the Computation Complexity}

If selecting the TLS-ESPRIT algorithm, the computational burden of the proposed method corresponds to the calculation the correlation matrix, Toeplitz matrix, the eigen-decomposition and pseudo-inverse operation. $X$ denotes the smoothing number of FOSS/FBSS, $\tau$ represents the delay time, which can be expressed by the number of sample points. Summing these processes, we can obtain the main computational complexity of these methods as Tab. 1 .

Compared with MTOEP method, our proposed TSMTOEP method has relatively less computational complexity. Meanwhile, it enhances the SNR, and further improves the DOA estimation performance at low SNR.

\section{Numerical Results}

In this section, we present several numerical examples to examine the effectiveness of the proposed TS-MTOEP method via comparison with the FOSS [5], FBSS [6], ESPRIT-like [9], FB-PTMR [11] and MTOEP [10]. We make comparisons based on the performance metrics including root-mean-square error (RMSE) and the probability of resolution (POR). The RMSE and POR are defined as

$$
\begin{aligned}
\operatorname{RMSE}_{\theta} & =\sqrt{\frac{1}{W P} \sum_{i=1}^{P} \sum_{w=1}^{W}\left(\tilde{\theta}_{i, w}-\theta\right)^{2}}, \\
\operatorname{POR}_{\theta} & =\left(\frac{1}{W P} \sum_{i=1}^{P} N_{i}\right) \times 100 \%
\end{aligned}
$$

where $W$ is the number of Monte Carlo trials, and $P$ is the signal source number. We set $\left|\hat{\theta}_{i, w}-\theta_{i}\right| \leq 2^{\circ}$ as an acceptable resolution and $N_{i}$ counts the number of a successful resolution for the $i$-th signal. A total of 2000 Monte Carlo trials are performed to obtain the statistic results in our examples, and there is a random phase delay from $[0,2 \pi]$ between these signals at each Monte Carlo trial. To

\begin{tabular}{|c|c|}
\hline Algorithm & Computational complexity \\
\hline FOSS/FBSS & $o\left((2 M+1)^{2} K+(2 M-X+2)^{3}+3(2 M-X+1) P^{2}+2 P^{3}\right)$ \\
\hline ESPRIT-Like & $o\left((2 M+1)^{2} K+(M+1)^{3}+3 M P^{2}+2 P^{3}\right)$ \\
\hline FB-PTMR & $o\left((2 M+1)^{2} K+(M+1)^{4}+(M+1)^{3}+3 M P^{2}+2 P^{3}\right)$ \\
\hline MTOEP & $o\left((2 M+1)^{2} K+(M+1)^{3}(2 M+1)+(M+1)^{3}+3 M P^{2}+2 P^{3}\right)$ \\
\hline TS-MTOEP & $o\left((2 M+1)^{2}(K-\tau)+(M+1)^{3}(2 M+1)+(M+1)^{3}+3 M P^{2}+2 P^{3}\right)$ \\
\hline
\end{tabular}

Tab. 1. Computational complexity of different methods. be fair, the ESPRIT method is also applied to find the DOAs for the FOSS, FBSS, ESPRIT-like, FB-PTMR and MTOEP schemes and the analysis is performed in Matlab.

\subsection{Performance versus SNR}

In the first example, we obtain the RMSE and POR performance as a function of the SNR. We assume that three narrowband far-field signal with carrier frequency $f=150 \mathrm{kHz}$ are received from $\left[-5^{\circ}, 4^{\circ}, 20^{\circ}\right]$ by an 11 -element, where $\left[-5^{\circ}, 4^{\circ}\right]$ are the coherent signals $\left(\beta_{2}=1\right.$, $\left.\varphi_{2}=0\right)$ and $20^{\circ}$ is the uncorrelated signal. The element is separated by half of the carrier wavelength $(d=\lambda / 2)$ as shown in Fig. 2. The noise is zero-mean, Gaussian distributed, and temporary white and the noise power $\sigma_{n}^{2}$ is adjust to give the desired $S N R=10 \log \left(\sigma_{n}^{-2}\right)$ [24]. The snapshot number is 200. The noise is the Gaussian white noise with zero mean, and the SNR varies from $-15 \mathrm{~dB}$ to $10 \mathrm{~dB}$.

In the ESPRIT-like algorithm, we choose the central row of the array output covariance matrix $\boldsymbol{R}_{\mathrm{yy}}$ to reconstruct the matrix $\boldsymbol{R}_{\mathrm{Y} i}, i=0$. The spatial smoothing number of the FOSS algorithm and FBSS algorithm is set to 4 . The time delay $\tau$ for our proposed method is set to 6 sample points. The simulation parameters defined as Tab. 2 .

From the simulation results shown in Fig. 2 and 3, when the coherent and uncorrelated signals exit, it can be clearly seen that the TS-MTOEP method achieves a much better DOA estimation performance. The FOSS and FBSS methods divide the whole array into overlapped subarrays, and average the subarray full rank covariance matrices to resolve the coherent signals, which leads to an effective decrease in aperture size and therefore a decrease in DOA estimation performance at low SNR. The MTOEP and FBPTMR methods exploit multi-rows of the array output covariance matrix to reconstruct the Toeplitz matrix and result in the noise energy is the square of input noise energy, which leads to degradation of the estimation performance in the low SNR. Meanwhile, compared with FBPTMR method, the MTOEP method does not introduce color noise, resulting in better DOA estimation performance. However, our proposed TS-MTOEP method takes advantage of the strong correlation of signal and the weak correlation of noise at different snapshots to construct the time-space correlation matrix, which enhances the noise suppression ability and improves the DOA estimation performance. Especially for $S N R=-10 \mathrm{~dB}$, our proposed method provides a much better estimation accuracy $\left(R M S E \approx 2^{\circ}\right)$ and resolution probability $\left(P O R \approx 85^{\circ}\right)$ than those of the other five methods ( $\left.R M S E \geq 7^{\circ}, P O R \leq 68^{\circ}\right)$.

\begin{tabular}{|c|c|c|c|l|c|}
\hline $\begin{array}{c}\text { Frequency } \\
{[\mathrm{kHz}]}\end{array}$ & $\begin{array}{c}\text { Snapshots } \\
{[\text { Sampling }} \\
\text { points }]\end{array}$ & $\begin{array}{c}\text { SNR } \\
{[\mathrm{dB}]}\end{array}$ & $\begin{array}{c}\text { Subarray } \\
\text { Number }\end{array}$ & $\begin{array}{l}\text { Time delay } \\
{[\text { Sampling }} \\
\text { points }]\end{array}$ & $\begin{array}{c}\text { DOA } \\
{\left[{ }^{\circ}\right]}\end{array}$ \\
\hline 150 & 200 & {$[-15,10]$} & 4 & 6 & {$[5,4,20]$} \\
\hline
\end{tabular}

Tab. 2. Simulation parameters. 


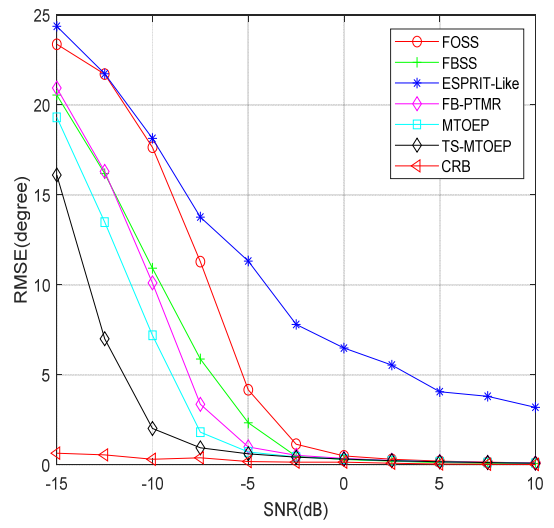

Fig. 2. RMSE vs. SNR at the number of snapshots 200.

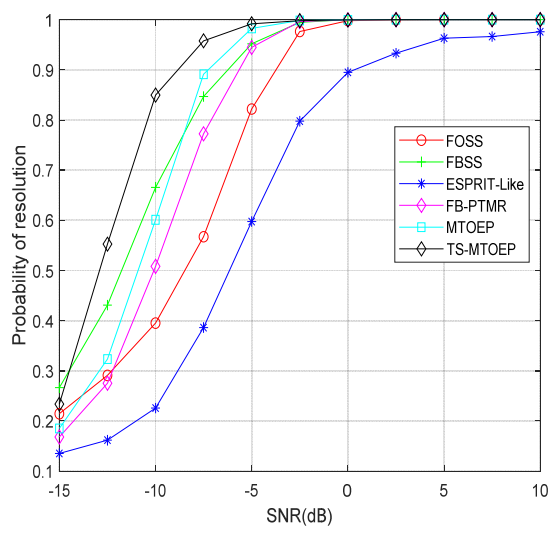

Fig. 3. POR vs. SNR at the number of snapshots 200 .

The simulation results from Fig. 2 and Fig. 3 also show that when the SNR decreases, our method is more close to $\mathrm{CRB}$. This is because the time-space processing enhances the SNR, which improves the DOA estimation performance in the low SNRs.

\subsection{Performance versus Snapshots}

In the second example, we examine the DOA estimation performance as a function of the number of snapshots at the low SNR value. The simulation conditions are the same as in Sec. 5.1, except that the snapshot number varies from 40 to 240 and the SNR is set to $-10 \mathrm{~dB}$.

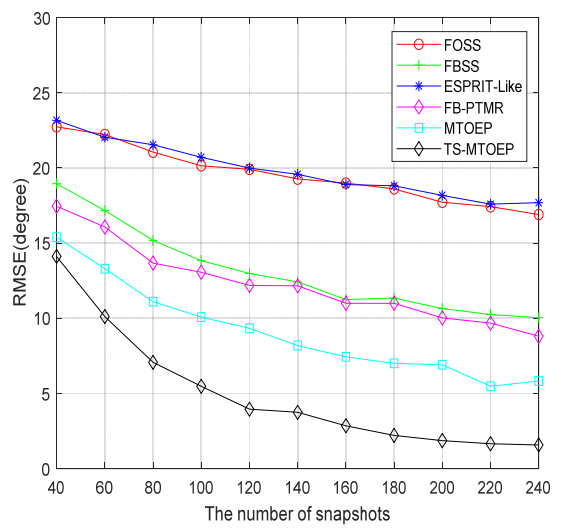

Fig. 4. RMSE vs. the number of snapshots at $S N R=-10 \mathrm{~dB}$.

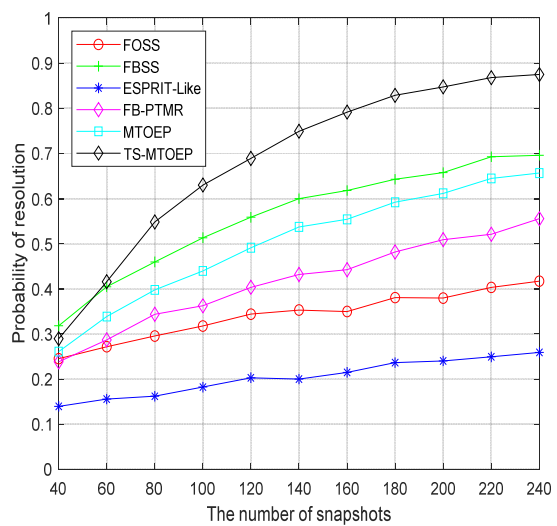

Fig. 5. POR vs. the number of snapshots at $S N R=-10 \mathrm{~dB}$.

Figure 4 and 5 illustrate that our proposed method achieves better estimation performance than those of the other methods when increasing the number of snapshots. This is because when the number of snapshots increased, it improves the estimation accuracy of the array output covariance matrix and the time-space correlation matrix, which further produces better DOA estimation performance. Compared with the Toeplitz reconstruction methods based on the array output covariance matrix, the TS-MTOEP method based on time-space correlation matrix enhances the noise suppression ability, and further improves the DOA estimation performance. Especially for a high number of snapshots such as 240, our proposed TS-MTOEP method achieves an improved DOA estimation accuracy $\left(R M S E \approx 2^{\circ}\right)$ and a higher probability of successful resolution $\left(P O R \approx 90^{\circ}\right)$ than the other methods $\left(R M S E \geq 6^{\circ}\right.$, $\left.P O R \leq 71^{\circ}\right)$.

\subsection{Performance versus Angular Separation}

In the third example, we obtain the RMSE and POR under the separation $\Delta \theta$ between the two coherent signals received from $\left[-5^{\circ},-5^{\circ},+\Delta \theta\right]$, where $\Delta \theta$ denotes angular separation which varies from $4^{\circ}$ to $18^{\circ}$. The SNR is $-10 \mathrm{~dB}$ and the snapshot number is 64 . The spatial smoothing number of the FOSS and FBSS algorithms is the same as in Sec. 5.2.

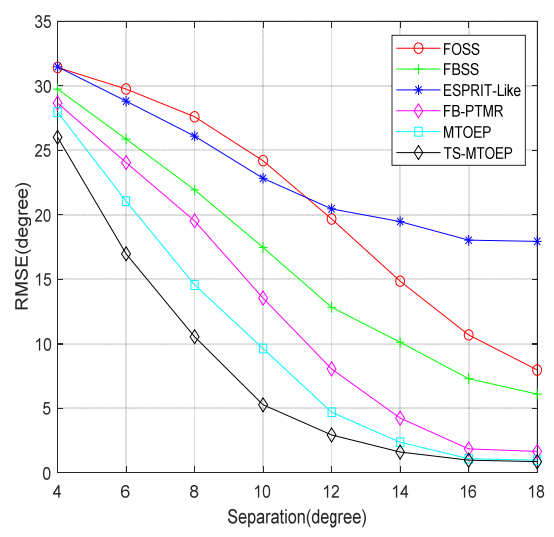

Fig. 6. RMSE vs. angular separation at $S N R=-10 \mathrm{~dB}$ 


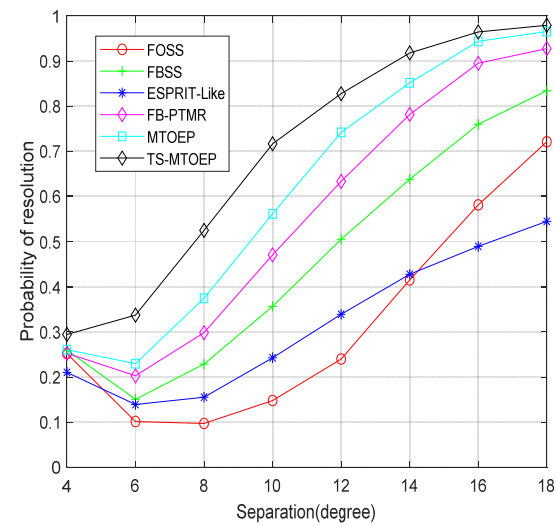

Fig. 7. POR vs. angular separation at $S N R=-10 \mathrm{~dB}$.

It can be seen in Fig. 6 and Fig. 7 that our proposed method TS-MTOEP method has the best RMSEs and PORs than that of the other methods across the entire angular separation range. This is because that the proposed method exploits all rows of the time-space correlation matrix to reconstruction the Toeplitz matrix, which improves the SNR and further provides better estimation performance. In particular, when the angular separation is $\Delta \theta=5^{\circ}$, our proposed TS-MTOEP method achieves better DOA estimation performance $\left(R M S E \approx 5^{\circ}, P O R \approx 70^{\circ}\right)$ than those from the other estimators (RMSE $\geq 9^{\circ}$, $\left.P O R \leq 58^{\circ}\right)$.

\section{Conclusions}

In this paper, we propose an improved MultipleToeplitz matrices reconstruction method called TSMTOEP. First, we construct the time-space correlation matrix by exploiting the strong correlation of signal and weak correlation of noise in time and space domains to improve the noise suppression ability. Then, the new Toeplitz matrix based on this time-space correlation matrix was reconstructed via MTOEP method. Finally, the DOAs are obtained by combining with the ESPRIT method. The numerical results show that our algorithm achieves better estimation performance than the existing Toeplitz matrix reconstruction methods in the sense of RMSE and POR. We would like to point out that our proposed method is also applicable to planar array, and it will be our future work.

\section{References}

[1] YUAN, L. T., JIANG, R. X., CHEN, Y. W. Gain and phase autocalibration of large uniform rectangular arrays for underwater 3-D sonar imaging systems. IEEE Journal of Oceanic Engineering, 2014, vol. 39, no. 3, p. 458-471. DOI: 10.1109/JOE.2013.2266195

[2] BONACCI, D., VINCENT, F., GIGLEUX, B. Robust DoA estimation in case of multipath environment for a sense and avoid airborne radar. IET Radar, Sonar \& Navigation, 2017, vol. 11, no. 5, p. 797-801. DOI: 10.1049 /iet-rsn.2016.0446
[3] SCHMIDT, R. D. Multiple emitter location and signal parameter estimation. IEEE Transactions on Antennas and Propagation, 1986, vol. 34, no. 3, p. 276-280. DOI: 10.1109/TAP.1986.1143830

[4] ROY, R., KAILATH, T. ESPRIT-estimation of signal parameters via rotational invariance techniques. IEEE Transactions on Acoustics, Speech, and Signal Processing, 1989, vol. 37, no. 7, p. 984-995. DOI: 10.1109/29.32276

[5] SHAN, T. J., WAX, M., KAILATH, T. On spatial smoothing for direction-of-arrival estimation of coherent signals. IEEE Transactions on Acoustics, Speech, and Signal Processing, 1985, vol. 33, no. 4, p. 806-811. DOI: 10.1109/tassp.1985.1164649

[6] PILlAI, S. U., KWON, B. H. Forward/backward spatial smoothing techniques for coherent signal identification. IEEE Transactions on Acoustics, Speech, and Signal Processing, 1989, vol. 37 , no. 1 , p. 8-15. DOI: 10.1109/29.17496

[7] BENGTSSON, M., OTTERSTEN, B. A generalization of weighted subspace fitting to full-rank models. IEEE Transactions on Signal Processing, 2001, vol. 49, no. 5, p. 1002-1012. DOI: $10.1109 / 78.917804$

[8] MAHNAZ, A. P., SEDIGHEH, G. M 1,2-MUSIC algorithm for DOA estimation of coherent sources. IET Signal Processing, 2017, vol. 11, no. 4, p. 429-436. DOI: 10.1049/iet-spr.2016.0246

[9] HAN, F. M., ZHANG, X. D. An ESPRIT-like algorithm for coherent DOA estimation. IEEE Antennas and Wireless Propagation Letters, 2005, vol. 4, no. 12, p. 443-446. DOI: 10.1109/LAWP.2005.860194

[10] ZHANG, W., HAN, Y., JIN, M., et al. Multiple-Toeplitz matrices reconstruction algorithm for DOA estimation of coherent signals. IEEE Access, 2019, vol. 7, p. 49504-49512. DOI: 10.1109/ACCESS.2019.2909783

[11] ZHANG, W., HAN, Y., JIN, M., et al. An improved ESPRIT-like algorithm for coherent signals DOA estimation. IEEE Communications Letters, 2020, vol. 24, no. 2, p. 339-343. DOI: 10.1109/L COMM.2019.2953851

[12] ZHENG, Z., FU, M., JIANG, D., et al. Localization of mixed farfield and near-field sources via cumulant matrix reconstruction. IEEE Sensors Journal, 2018, vol. 18, no. 18, p. 7671-7680. DOI: 10.1109/JSEN.2018.2863749

[13] LI, X., GONG, Q., ZHONG, S., et al. Near-field noncircular sources localization based on fourth-order cumulant. IEEE Access, 2020, vol. 8, p. 120575-120585. DOI: 10.1109/ACCESS.2020.3006292

[14] CHENG, G. H., ZENG, X. P., JIAO, S., et al. High accuracy nearfield localization algorithm at low SNR using fourth-order cumulant. IEEE Communications Letters, 2020, vol. 24, no. 3, p. 553-557. DOI: 10.1109/LCOMM.2019.2959576

[15] MA, X., DONG, X., XIE, Y. An improved spatial differencing method for DOA estimation with the coexistence of uncorrelated and coherent signals. IEEE Sensors Journal, 2016, vol. 16, no. 10 , p. 3719-3723. DOI: 10.1109/JSEN.2016.2532929

[16] LIU, G., SUN, X. Spatial differencing method for mixed far-field and near-field sources localization. IEEE Signal Processing Letters, 2014, vol. 21, no. 11, p. 1331-1335. DOI: 10.1109/LSP.2014.2326173

[17] SHI, J., HU, G., ZHANG, X. Direction of arrival estimation in low-grazing angle: A partial spatial-differencing approach. IEEE Access, 2017, vol. 5, p. 9973-9980. DOI: 10.1109/ACCESS.2017.2706193

[18] ZHANG, Y. M., MA, W. F., AMIN, M. G. Subspace analysis of spatial time-frequency distribution matrices. IEEE Transactions on Signal Processing, 2001, vol. 49, no. 4, p. 747-759. DOI: $10.1109 / 78.912919$ 
[19] LIN, J. C., MA, X. C., YAN, S. F., et al. Time-frequency multiinvariance ESPRIT for DOA estimation. IEEE Antennas and Wireless Propagation Letters, 2016, vol. 15, p. 770-773. DOI: 10.1109/LAWP.2015.2473664

[20] ZHANG, H. J., BI, G. A., CAI, Y. L., et al. DOA estimation of closely-spaced and spectrally-overlapped sources using a STFTbased MUSIC algorithm. Digital Signal Processing, 2016, vol. 52, no. C, p. 25-34. DOI: 10.1016/j.dsp.2016.01.015

[21] DAI, X. R., ZHANG, X. F., WANG, Y. F. Extended DOA-matrix method for DOA estimation via two parallel linear arrays. IEEE Communications Letters, 2019, vol. 23, no. 11, p. 1981-1984. DOI: 10.1109/LCOMM.2019.2939245

[22] ZHENG, Z., MU, S. L. Two-dimensional DOA estimation using two parallel nested arrays. IEEE Communications Letters, 2020, vol. 24, no. 3, p. 568-571. DOI: 10.1109/LCOMM.2019.2958903

[23] KRIM, H., VIBERG. M. Two decades of array signal processing research: The parametric approach. IEEE Signal Processing Magazine, 1996, vol. 13, no. 4, p. 67-94. DOI: 10.1109/79.526899
[24] BELOUCHRANI, A., AMIN, M. G. Time-frequency MUSIC. IEEE Signal Processing Letters, 1999, vol. 6, no. 5, p. 109-110. DOI: 10.110 9/97.755429

\section{About the Authors ...}

Bingbing QI (corresponding author) received the B.S. degree from Yanshan University, Hebei, China, in 2010, and the M.S. degree from the Yanshan University, Hebei, China, in 2013. Currently, he is pursuing the Ph.D. degree in Communication and Information Systems with the Nanjing University of Aeronautics and Astronautics, Nanjing, China. His research interests include radar, sonar and array signal processing.

Wei LI was born in Nei Mongol in 1990. He received his M.Sc. from Harbin Engineering University. His research interests include sonar and array signal processing. 